\title{
Impact of relative and absolute values on selective attention
}

\author{
Sunghyun $\mathrm{Kim}^{1}$ • Melissa R. Beck ${ }^{1}$
}

Published online: 7 April 2020

(C) The Psychonomic Society, Inc. 2020

\begin{abstract}
Valuable stimuli receive attentional priority. However, it is unknown whether the mechanism of the attentional priority is based on relative (e.g., higher) or absolute (e.g., 45 points) values. Therefore, we manipulated the relative and absolute values independently in a modified value-driven attentional capture paradigm. In the training phase, where associative learning occurs between color and reward value, two test target colors were each presented with another different target color (reference target colors) in separate context blocks. Therefore, each test target color had different reference points. In the test phase, the two test target colors were used as singleton distractor colors. In the training phase of Experiment 1, the absolute reward value of the test target colors was the same, but one had a higher value than its reference target color and the other had a lower value. In the test phase, the high relative value color distractor captured attention more, suggesting that the relative value of stimuli influenced selective attention. In Experiment 2 the relative value of the test target colors was the same, but the absolute value was higher for one. The high and low absolute value color distractors captured attention equally in the test phase, indicating little impact of the absolute value on selective attention. These findings suggest that the relative value, rather than absolute value, plays a critical role in the allocation of attention. Accordingly, the present study suggests that prospect theory (Kahneman \& Tversky, Econometrica, 47 (2), 363-391, 1979) can be extended to earlier cognitive stages such as selective attention.
\end{abstract}

Keywords Visual selective attention $\cdot$ Prospect theory $\cdot$ Value-driven attentional capture $\cdot$ Reference dependence

\section{Introduction}

Only a limited amount of information can be attended to at a given time (Broadbent, 1958). A growing body of evidence shows that the value of an object can influence attentional allocation: value-driven attentional capture (Anderson, Laurent, \& Yantis, 2011; Chelazzi, Perlato, Santandrea, \& Della Libera, 2013; Lee \& Shomstein, 2014). However, there are two types of value, relative value and absolute value. The

Public Significance Statements: A visual stimulus that is associated with reward attracts visual attention more readily than other stimuli. The mechanism by which valuable objects capture visual attention was explored. The present study suggests that value-driven attentional capture is on the basis of the relative value of the objects rather than the absolute value. That is, visual stimuli that capture attention do so because they are more valuable compared to other stimuli and not because of the amount of the value itself.

Sunghyun Kim

skim58@1su.edu

1 B5 Audubon, Department of Psychology, Louisiana State University, Baton Rouge, LA 70803, USA relative value of an object is dependent on the values of the other available objects in the same context (e.g., higher or lower), whereas the absolute value of an object is not influenced by the values of other available objects (e.g., 50 points). Critically, both types of values are represented separately and simultaneously in the brain (Grabenhorst \& Rolls, 2009). However, this does not necessarily mean that the relative and absolute values equally influence human behaviors (e.g., Kahneman \& Tversky, 1979). Thus, the present study explored whether the relative and/or absolute value associated with an object determines if the object will attract attention.

Prospect theory (Kahneman \& Tversky, 1979) suggests that relative rather than absolute value influences behaviors in later cognitive stages such as judgement and decision making (the reference-dependence aspect of prospect theory). According to prospect theory, the value of an object is determined by a reference point for the object. For example, when it is possible to gain either 1 or 10 dollars, receiving 10 dollars will give rise to satisfaction, but when it is possible to gain either 10 or 20 dollars, receiving 10 dollars will lead to disappointment. In this case, the reference point of either 1 dollar or 20 dollars determines the amount of satisfaction in receiving 10 dollars. The critical role of the relative value on judgement 
and decision making has been extensively demonstrated in behavioral economics (for a review, see Barberis, 2013).

It is, however, unclear whether relative rather than absolute value (the reference-dependence aspect of prospect theory) affects earlier cognitive stages, such as selective attention, because research on prospect theory has been focused on the later cognitive stages like judgement and decision making. Another aspect of prospect theory (the weighting function of prospect theory) has been shown to extend to earlier cognitive stages (Vincent, 2011). Specifically, during visual search, the probability of a search target appearing at a particular location was overweighted when the probability was low and underweighted when it was high. This pattern is consistent with the weighting function of prospect theory (Kahneman \& Tversky, 1979), alluding to the extendibility of prospect theory to selective attention. Thus, the current research investigates the reference-dependence aspect of prospect theory in selective attention. This step is critical to determining the applicability of prospect theory to selective attention because reference dependence is considered the key premise of prospect theory (Bendor, 2004).

The current study tests the applicability of the reference-dependence aspect of prospect theory to selective attention by modifying the value-driven attentional capture paradigm to independently manipulate the relative and absolute values. Previous studies on value-driven attentional capture could not determine whether valuable stimuli capturing attention was due to the relative or absolute value of the stimuli because the valuable stimuli were not only relatively but also absolutely more valuable (e.g., Anderson, Laurent, \& Yantis, 2011; Bucker \& Theeuwes, 2017; Le Pelley, Pearson, Porter, Yee, \& Luque, 2019; MacLean \& Giesbrecht, 2015; Mine \& Saiki, 2015; Roper, Vecera, \& Vaidya, 2014). Therefore, we needed to modify the typical methodology used in previous value-driven attentional capture literature such that the absolute value and the relative value could be independently manipulated.

In a typical value-driven attentional capture study, associations of value for stimuli are learned during a training phase and then a test phase reveals that stimuli previously associated with a higher reward value receive attentional priority compared to stimuli previously associated with a lower reward value (Anderson et al., 2011; Bucker \& Theeuwes, 2017; Mine \& Saiki, 2015). For instance, the Anderson et al. (2011) study included a training phase in which the associative learning between color and reward value occurred. Participants searched for red and green circles amongst different color circles, and one of these target colors was presented on each trial. Critically, in the training phase, higher rewards were given more often when the target was red ( 5 cents for $80 \%$ of the trials and 1 cent for $20 \%$ of the trials) compared to when it was green ( 1 cent for $80 \%$ of the trials and 5 cents for
$20 \%$ of the trials). Accordingly, the absolute value was higher for red than for green. In the test phase, the high-valued color captured attention more than the low-valued color. The targetdefining feature during the test phase was a unique shape instead of color (a white circle among white diamonds or a white diamond among white circles). Critically, on some trials, one of the distractors was red (high valued color) or green (low valued color) equally often: color singleton distractor. Although there was no benefit in attending to the color singleton distractors (i.e., color was task-irrelevant and reward was no longer given), responses were slower when the singleton distractor was the high-valued color (red) than when it was the low-valued color (green). This finding led the authors to conclude that stimuli previously associated with a higher value captured attention more (Anderson et al., 2011).

This typical methodology (e.g., Anderson et al., 2011) of value-driven attentional capture cannot reveal whether the mechanism by which value modulates selective attention is based on relative or absolute value. This is because the high reward associated color had a higher absolute and a higher relative value than the low reward-associated color in the training phase. Specifically, both the higher and the lower valued colors were target colors in the same context (the training phase): one of the target colors was randomly chosen to be presented on each trial in the training phase. Therefore, both target colors were always task relevant throughout the training phase, allowing each color to become a reference point for the other to compare their reward values. In line with this, Anderson (2016) mentioned that the relative and absolute values had not been independently manipulated in a single experiment.

Anderson (2016), however, stated that relative rather than absolute value seemed to influence value-driven attentional capture. The idea remained unsolved because it is obtained from suggestive evidence across multiple studies. Accordingly, the present study directly tested the impacts of the relative and absolute values on value-driven attentional capture through the independent manipulation of relative and absolute values in a single experiment.

To independently manipulate relative and absolute values, the present experiments adapted reference dependence (Kahneman \& Tversky, 1979) into the classic value-driven attentional capture paradigm (Anderson et al., 2011). In the training phase, there were two separate contexts, so that each of the test target colors (red and green) that were used as valueassociated distractor colors in the test phase had a different reference target color (yellow, blue) during the training phase. Accordingly, the use of separate contexts permitted the independent manipulation of relative and absolute values. Experiment 1 examined the impact of the relative value while controlling the absolute value. Experiment 2 examined the impact of the absolute value while controlling the relative value. 


\section{Experiment 1}

To explore the impact of relative value on selective attention, on the basis of the Anderson et al.'s (2011) paradigm, we made the absolute reward values of red and green the same and the relative reward values different by providing the associative learning of value for red and green in separate context blocks (see Table 1). For example, in blocks 1, 3, and 5 of the training phase, target colors were red and yellow, and red targets were more likely to give a higher reward than yellow targets. In blocks 2,4 , and 6 , target colors were green and blue, and green targets were more likely to give a lower reward than blue targets. Accordingly, while the absolute value of red and green was the same, the relative value was higher for red and lower for green due to their different reference points (yellow and blue associated values respectively). In the test phase, as in Anderson et al. (2011), the target was a unique shape, and on some trials either a red or a green singleton distractor was presented (see Fig. 1). If the relative value influences selective attention, search will be slower when the target appears with the high than when it appears with the low relative value color distractor.

\section{Method}

Participants Fifty-six undergraduate students with normal or corrected-to normal vision participated for course credit. The sample size was determined from value-driven effect sizes in Anderson et al. (2011). The critical analysis to assess valuedriven attentional capture was a comparison between the highand low-value color distractors in the test phase. G power (power $=0.90$, alpha $=0.05$, and Cohen's $\mathrm{D}=0.81$ ) showed that at least 19 participants were necessary. However, the number of training trials (504 trials) for each color in Anderson et al. was around three times of those (180 trials) in the current study, so we aimed for three times the sample size. a
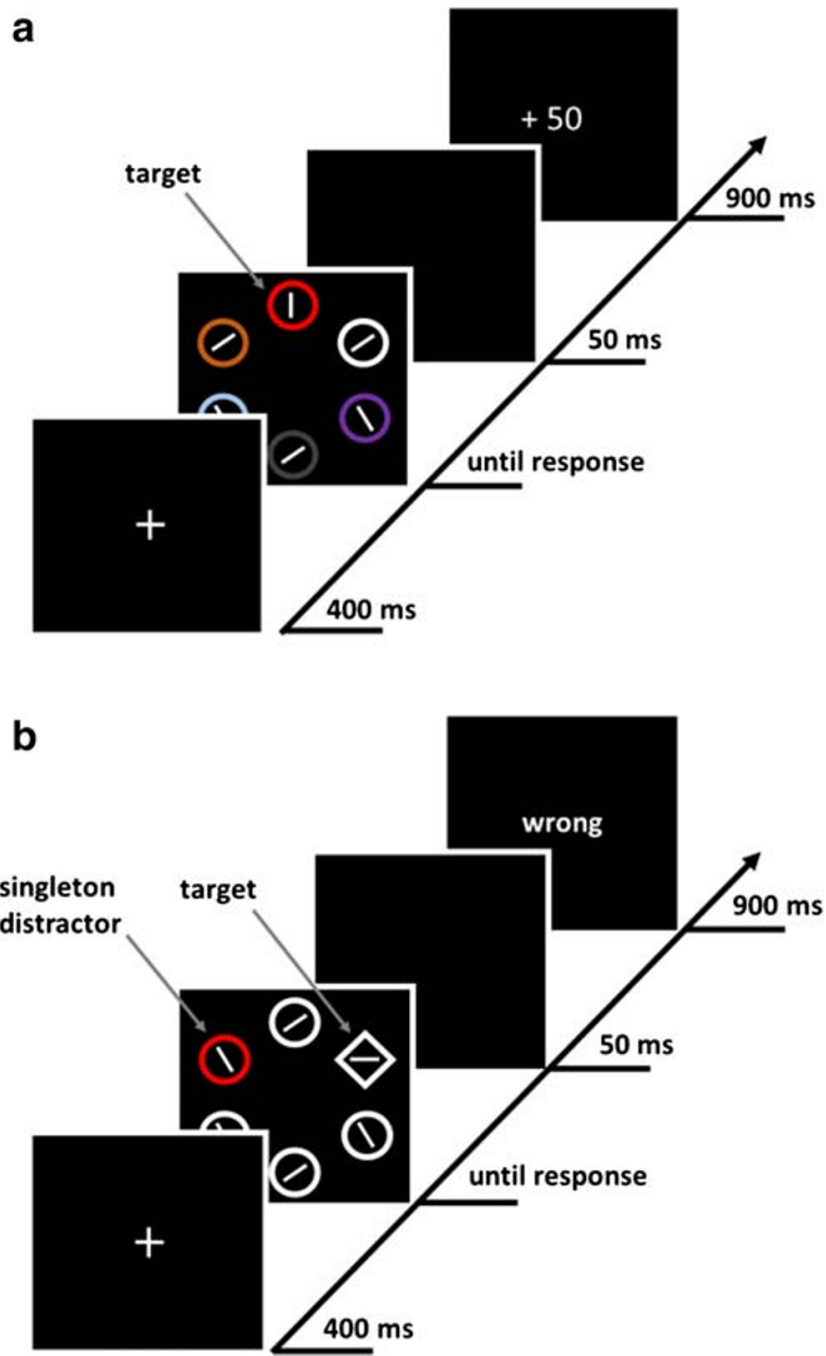

Fig. 1 Sequence of trial events of the training phase (A) and the test phase (B) in Experiments 1 and 2

Apparatus and stimuli Stimuli were presented on a 20 -in. monitor. The distance between the participants and the monitor was approximately $60 \mathrm{~cm}$ but was not constrained.

Table 1. Experiment 1 training phase: Relative value

\begin{tabular}{llllll}
\hline Reward points & \multicolumn{1}{l}{ Block } & & & \\
\cline { 2 - 6 } & 1 & 2 & 3 & 4 & 5 \\
\hline $50(90 \%)$ & Red (high) & Green (low) & Red (high) & Green (low) & Red (high) \\
$1(10 \%)$ & Yellow & & Yellow & Green (low) \\
$1(90 \%)$ & & Blue & & Blue & Blue \\
$50(10 \%)$ & & & & & \\
$100(90 \%)$ & & & & & \\
\hline
\end{tabular}

Note. In this example, target colors are red and yellow in the 1,3, and 5 context blocks and green and blue in the 2, 4, and 6 context blocks. The absolute value of the red and green is the same (50 points on $90 \%$ of trials, 1 point on $10 \%$ of trials), whereas the relative value of them is different (red is relatively higher compared to yellow (a reference point of red) and green is relatively lower compared to blue (a reference point of green)). In the test phase, singleton color distractor is red (high relative value in the training) or green (low relative value in the training). The test target colors (red and green), the reference target colors (yellow and blue), and the block order were fully counterbalanced across 56 participants 
In the training phase, each trial consisted of fixation, search, blank, and feedback displays (see Fig. 1). The background of the screen was black for all displays. In the fixation display, a white cross bar was presented in the center of the screen. In the search display, six circles $\left(1.4^{\circ}\right.$ diameter each $)$ were presented around an invisible circle ( $5^{\circ}$ radius). Inside a target object, a horizontal or vertical white line was presented, and inside each distractor object, a white line tilted $45^{\circ}$ to the left or right was presented. One of the six circles was a target color (yellow, red, green, or blue) and the others were distractor colors (orange, purple, aqua, white, and gray). In the search display of the test phase (see Fig. 1), the search target was a unique shape: a circle among diamonds or a diamond among circles.

Design The experiment consisted of 720 training trials followed by 384 test trials (see Table 1). The relative value (high, low) of the two test colors (red and green) was manipulated within three alternating blocks of the training phase. Correct responses earned 50 points on $90 \%$ of trials and 1 point on $10 \%$ of trials for the test target colors (red and green). For the reference target colors, correct response earned 100 points on $90 \%$ of trails and 1 point on $10 \%$ of trials for one of the reference target colors (blue and yellow), and 1 point on $90 \%$ of trials and 50 points on $10 \%$ of trials for the other. The test target color that was paired with the reference target color receiving 100 points (90\%) and 1 point (10\%) in the training blocks was the low relative value target color. The test target color paired with the reference target color receiving 1 point $(90 \%)$ and 50 points (10\%) in the training blocks was the high relative value target color. Within each block, each of the two target colors (one test target color and one reference target color) were presented on $50 \%$ of trials.

The test target colors (red or green), the reference target colors (yellow or blue), and the block order were fully counterbalanced across the participants. Specifically, in 1, 3 , and 5 training blocks, the target colors were a test target color (either red or green) and a reference target color (either blue or yellow). In 2, 4, and 6 training blocks, the target colors were the two remaining colors (e.g., if red and yellow in 1, 3, and 5 blocks, then green and blue in 2, 4, and 6 blocks).

Procedure In the training phase, participants were instructed to find a circle with one of the two target colors and report the orientation of the line inside the circle by pressing the N-key for a horizontal line or M-key for a vertical line as quickly and accurately as possible. On each trial, the fixation display was presented for $400 \mathrm{~ms}$, followed by the search display until a response was made. After the response, there was a blank display for $50 \mathrm{~ms}$ and then a feedback display for $900 \mathrm{~ms}$. In the feedback display, earned points (e.g., +50 ) were presented when a correct response was made within 1,500 ms. For incorrect responses, "+ 0 (wrong)" was presented. For slow responses (over $1500 \mathrm{~ms}$ ), "+ 0 (too slow)" was presented.

Participants first completed 40 practice trials during the training phase. In the first 20 practice trials, the target colors were the same as the target colors in the first, third, and fifth training blocks (e.g., red and yellow). In the second 20 practice trials, the target colors were the same as those of second, fourth, and sixth training blocks (e.g., green and blue). Before each practice, oral and written instructions regarding the target colors were provided. Before each of the six training blocks, written instructions regarding the target colors was provided. Participants were informed before that they would receive points when fast and correct responses were made, and the experiment would finish earlier as they received more points. However, unbeknownst to the participants, earned points did not affect the number of trials in the experiment.

The test phase followed immediately after the training phase, participants were instructed to search for a unique shape (a circle among diamonds or a diamond among circles) and report the orientation of the line in the unique shape; therefore, color was task-irrelevant. Also, they were informed that reward points were not given in the test phase. The timing of each screen and required response was the same as in the training phase, but the search display was replaced with the shape singleton search display (see Fig. 1). During 20 practice trials, an experimenter checked and confirmed that participants understood the singleton shape detection instructions. Then, 384 randomly ordered test trials were given. On 96 of the 384 trials, one of the non-target objects was green. On another 96 trials, one of the non-target objects was red. On the remaining 192 trials, all objects were white.

\section{Results and discussion}

The dependent variable was response time (RT) recorded from the onset of the search display. Only correct responses were included in analyses of RTs (incorrect trials: $4.6 \%$ in training phase, $8.6 \%$ in test phase). Also, trials in which RT was shorter than $150 \mathrm{~ms}$ ( $0 \%$ in training phase, $0.1 \%$ in test phase) or longer than $1,500 \mathrm{~ms}(0.9 \%$ in training phase, $5.6 \%$ in test phase) were excluded from the analysis. The first three trials of each block in the training phase and of the test phase were also excluded from the analysis to allow some time to change the attentional control settings.

Training phase RT was not different between when the target was relatively high-valued color (mean $=668 \mathrm{~ms}$, standard error $=9 \mathrm{~ms}$ ) and low-valued color (mean $=668 \mathrm{~ms}$, standard error $=10 \mathrm{~ms}), t(55)=.01, p=.99$.

Test phase RT was shorter when no color singleton distractor was presented $(790 \mathrm{~ms})$ than when the high- $(866 \mathrm{~ms})$ and low- $(854 \mathrm{~ms})$ valued color singleton distractors were 
presented, $p s<.001$. Importantly, RT was slower when the high relative value color distractor was presented than when the low relative value color distractor was presented, $t(55)=$ 2.28, $p=.027, d=.30$ (see Fig. 2), suggesting that the high relative value color distractor captured attention more than the low relative value color distractor. This effect was not due to the speed-accuracy trade-off, given that accuracy was not different between the low $(91.48 \%)$ and high $(91.25 \%)$ relative value color distractors, $t(55)=.39, p=.69$. This implies that the high relative value color distractor captured attention more than the low relative value color distractor. Accordingly, relative value influenced selective attention.

\section{Experiment 2}

The purpose of Experiment 2 was to examine if the absolute value influences value-driven attentional capture. Therefore, while the relative value of red (higher) and green (higher) was the same, their absolute value was different (see Table 2). If absolute value impacts selective attention, the high absolute value distractor would capture attention more than the lowvalued distractor in the test phase.

\section{Method}

Participants Fifty-six undergraduate students with normal or corrected-to normal vision participated for course credit.

Apparatus, stimuli, design, and procedure The apparatus, stimuli, design, and procedure were identical to Experiment 1. The only difference was the reward-point allocation during training. In the training phase, one test target color (e.g., red) gave 50 points for $90 \%$ of the trials and 1 point for $10 \%$ of the trials, and the other test target color (e.g., green) gave 100 points for $90 \%$ of the trials and 1 point for $10 \%$ of the trials (see Table 2). One of the reference targets (e.g., yellow) gave 1 point for $90 \%$ of the trials and 50 points for $10 \%$ of the trials and the other reference target (e.g., blue) gave 50 points for $90 \%$ of the trials and 1 point for $10 \%$ of the trials.

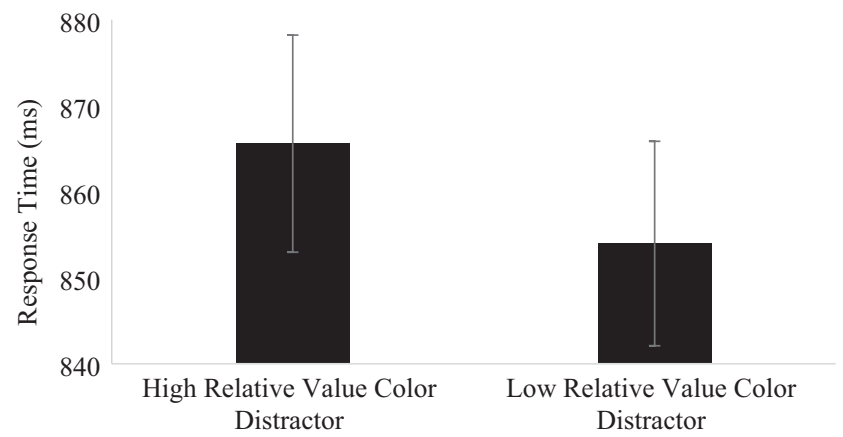

Fig. 2 Mean of response times in the test phase of Experiment 1. Error bars represent standard error of the mean

\section{Results and discussion}

The following trials were excluded in the analyses: incorrect trials $(4.5 \%$ in training phase, $7.3 \%$ in test phase), trials with RTs shorter than $150 \mathrm{~ms}(0 \%$ in training phase, $0.07 \%$ in test phase) or longer than $1,500 \mathrm{~ms}(0.7 \%$ in training phase, $4.3 \%$ in test phase), and the first three trials of each block in the training phase and the test phase.

Training phase RT was not different between the high absolute value color target $($ mean $=676 \mathrm{~ms}$, standard error $=12 \mathrm{~ms})$ and low absolute value color target (mean $=676 \mathrm{~ms}$, standard error $=11 \mathrm{~ms}), t(55)=.03, p=.97$.

Test phase As in Experiment 1, RT was shorter when no color singleton distractor was presented $(789 \mathrm{~ms})$ than when the high- $(863 \mathrm{~ms})$ and low- $(860 \mathrm{~ms})$ valued color singleton distractors were presented, $p s<.001$. Critically, RT was not different between when the singleton distractor was the high absolute value color and the low absolute value color (see Fig. $3), t(55)=-.86, p=.39, d=-.11$, suggesting that the high absolute value color distractor did not capture attention more than the low absolute color distractor. The lack of RT difference was not due to the speed-accuracy trade-off, given no difference between the low (92.73\%) and high (92.59\%) absolute value color distractors, $t(55)=.29, p=.77$. Additionally, the Bayes factor $\left(\mathrm{BF}_{+0}\right)=5.4$ (van Doorn et al., 2019), indicating that the prediction of more attentional capture by the high than low absolute value color distractor was 5.4 times less favored than the null. Accordingly, there is no evidence of an impact of absolute value on selective attention.

To directly compare the impacts of the relative and absolute values on the value-driven attentional capture, data between Experiments 1 and 2 were compared. The mixed ANOVA on color (high- and low-valued color singleton distractor) as a within-subject factor and type of value (relative and absolute) as a between-subjects factor revealed an insignificant main effect of color, $F(1,110)=1.43, p=.24, \eta_{p}^{2}=.01$. Critically, the interaction was significant, $F(1,110)=5.30, p=.02, \eta_{p}^{2}=$ .05 , supporting a stronger impact of relative than absolute value on selective attention.

\section{General discussion}

The present study showed that the high relative reward value color distractor captured attention more than the low relative reward value color distractor when the absolute value of them was the same (Experiment 1), indicating that the relative value influenced the allocation of attention. However, the high absolute reward value color distractor did not capture attention 
Table 2. Experiment 2 training phase: Absolute value

\begin{tabular}{|c|c|c|c|c|c|c|}
\hline \multirow[t]{2}{*}{ Reward points } & \multicolumn{6}{|l|}{ Block } \\
\hline & 1 & 2 & 3 & 4 & 5 & 6 \\
\hline $\begin{array}{l}50(90 \%) \\
1(10 \%)\end{array}$ & Red (low) & & Red (low) & & Red (low) & \\
\hline $\begin{array}{l}100(90 \%) \\
1(10 \%)\end{array}$ & & Green (high) & & Green (high) & & Green (high) \\
\hline $\begin{array}{l}1(90 \%) \\
50(10 \%)\end{array}$ & Yellow & & Yellow & & Yellow & \\
\hline $\begin{array}{l}50(90 \%) \\
1(10 \%)\end{array}$ & & Blue & & Blue & & Blue \\
\hline
\end{tabular}

Note. In this example, target colors are red and yellow in the 1,3, and 5 context blocks and green and blue in the 2, 4, and 6 context blocks. The relative value of the red and green is the same, whereas the absolute value of them is different (Red: 50 points on $90 \%$ of trials and 1 point on $10 \%$ of trials, Green: 100 points on $90 \%$ of trials and 1 point on $10 \%$ of trials). In the test phase, singleton color distractor is red (low absolute value in the training) or green (high absolute value in the training). The test target colors (red and green), the reference target colors (yellow and blue), and the block order were fully counterbalanced across 56 participants

more than the low absolute reward value color distractor when the relative value of them was the same (Experiment 2), indicating little impact of the absolute value on the allocation of attention. This is the first study in the value-driven attentional capture literature to test the impacts of absolute and relative values through the independent manipulation of absolute and relative values and to confirm the critical role of relative value in value-driven attentional capture.

The present study demonstrates that relative value is learned within an immediate context rather than a general or broad context. The context in which the relative value of colors was determined was specific to blocks in which the colors were presented, rather than the whole training session. This was because relative value is set by a reference point (Kahneman \& Tversky, 1979), and in the present study a reference point of the red and green was within the blocks where the colors were presented as targets. For example, in block 1 of Table 1, targets are red and yellow. Accordingly, participants search for both colors during block 1, allowing each of them to be a reference point for the other to compare their values. In block 2, however, targets are green and blue, but neither red nor yellow. Therefore, it is unlikely that participants unnecessarily think about red and yellow in block 2

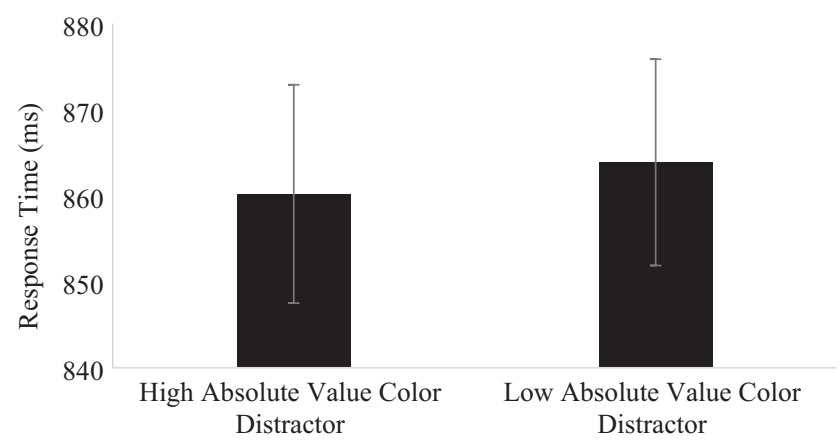

Fig. 3 Mean of response times in the test phase of Experiment 2. Error bars represent standard error of the mean because these colors are non-target colors. Likewise, in block 1 , participants would not search for green and blue because these colors are not task relevant during block 1. Thus, the context for value-driven attentional capture operates within a set of trials where a color is task relevant, and the context used to establish the relative value does not extend beyond to trials where the color is no longer task relevant.

In line with Vincent (2011), the present study showed that prospect theory can extend to early cognitive stages of processing. However, while Vincent (2011) addressed the weighting function of prospect theory, the current research investigated the reference dependence in the value function of prospect theory. According to reference dependence (Kahneman \& Tversky, 1979), the subjective value depends on a reference point so that the relative value of an object compared to its reference's value is critical. In line with the reference dependence, the current study showed that the value-driven attentional capture was stronger for the color whose value was larger compared to the reference color's value than the color whose value was smaller compared to the reference color's value.

The impact of relative value for reward aligns with the importance of context in the value-driven attentional capture effect. Value-driven attentional capture relies on the context in which the stimuli-reward associative learning occurs. Specifically, a stimulus captures attention in a background scene where the stimulus was previously valuable more than in another scene where the stimulus was not (scene contexts; Anderson, 2015). Furthermore, predictive relationships between stimuli and reward are necessary for value-driven attentional capture (predictability contexts; Sali, Anderson, \& Yantis, 2014), because dopaminergic prediction errors, calculated in the predictive relationships, serve as teaching signals that establish value-driven attentional capture (Anderson, 2019). In line with the importance of context in these previous 
findings, the present study showed the importance of contexts for the reward value. Value-driven attentional capture occurs on the basis of relative value, which is determined by a reference point. A reference point can change depending on contexts (reference dependence contexts; Kahneman \& Tversky, 1979).

A critical role of a relative property across different cognitive stages has been found in other research, such as relational theory. According to relational theory (Becker et al., 2010; Goldstein \& Beck, 2016), an attention control setting is established depending on a relation between a target and distractor (a reference) near to the target. For example, when an orange target is presented with yellow distractors, an attentional control setting is adjusted to "redder" (a relative property) rather than to an "orange" color (an absolute property), suggesting that a relative reference not involving reward values can influence selective attention. Although the references in relational theory do not involve reward values, relational theory is consistent with the current study and prospect theory in that relative rather than absolute properties are critical in cognitive processes.

In conclusion, the present study contributes to the understanding of value-driven attentional capture and prospect theory. The mechanism of valuable stimuli receiving attentional priority is on the basis of the relative values of the reward. Reference dependence, the key premise of prospect theory, operates in the earlier cognitive stage, selective attention. Future research may allow generalization to tasks using monetary rewards because the absolute value of real money may be more salient than non-monetary rewards, and prospect theory was demonstrated using both monetary and non-monetary outcomes (Tversky \& Kahneman, 1981).

Open Practices Statement The data from both experiments and the preregistered document (As-Predicted) for Experiment 1 are available at the Center for Open Science: https://osf.io/umd8c/

\section{References}

Anderson, B. A. (2016). The attention habit: How reward learning shapes attentional selection. Annals of the new York Academy of Sciences, $1369(1), 24-39$.

Anderson, B. A. (2015). Value-driven attentional priority is context specific. Psychonomic Bulletin \& Review, 22(3), 750-756.

Anderson, B. A. (2019). Neurobiology of value-driven attention. Current opinion in psychology, 29, 27-33.
Anderson, B. A., Laurent, P. A., \& Yantis, S. (2011). Learned value magnifies salience-based attentional capture. PloS one, 6(11), e27926.

Barberis, N. C. (2013). Thirty years of prospect theory in economics: A review and assessment. Journal of Economic Perspectives, 27(1), 173-96.

Becker, S. I., Folk, C. L., \& Remington, R. W. (2010). The role of relational information in contingent capture. Journal of Experimental Psychology: Human Perception and Performance, 36, 1460-1476.

Bendor, J. (2004). Bounded rationality. In N. J. Smelser \& P. B. Baltes (Eds.), International encyclopedia of the social and behavioral sciences Vol. 2 (pp. 1303-1307). Amsterdam, The Netherlands: Elsevier

Broadbent, D. E. (1958). Perception and communication. New York: Oxford University Press.

Bucker, B., \& Theeuwes, J. (2017). Pavlovian reward learning underlies value driven attentional capture. Attention, Perception, \& Psychophysics, 79(2), 415-428.

Chelazzi, L., Perlato, A., Santandrea, E., \& Della Libera, C. (2013). Rewards teach visual selective attention. Vision research, 85, 58-72.

Goldstein, R.R. \& Beck, M. R. (2016). Inattentional blindness: A combination of a relational set and a feature inhibition set? Attention, Perception, \& Psychophysics, 78, 1245-1254.

Grabenhorst, F., \& Rolls, E. T. (2009). Different representations of relative and absolute subjective value in the human brain. Neuroimage, 48(1), 258-268.

Kahneman, D., \& Tversky, A. (1979). Prospect theory: An analysis of decision under risk. Econometrica, 47(2), 363-391.

Lee, J., \& Shomstein, S. (2014). Reward-based transfer from bottom-up to top-down search tasks. Psychological Science, 25(2), 466-475.

Le Pelley, M. E., Pearson, D., Porter, A., Yee, H., \& Luque, D. (2019). Oculomotor capture is influenced by expected reward value but (maybe) not predictiveness. Quarterly Journal of Experimental Psychology, 72(2), 168-181.

MacLean, M. H., \& Giesbrecht, B. (2015). Irrelevant reward and selection histories have different influences on task-relevant attentional selection. Attention, Perception, \& Psychophysics, 77(5), 15151528.

Mine, C., \& Saiki, J. (2015). Task-irrelevant stimulus-reward association induces value-driven attentional capture. Attention, Perception, \& Psychophysics, 77(6), 1896-1907.

Roper, Z. J., Vecera, S. P., \& Vaidya, J. G. (2014). Value-driven attentional capture in adolescence. Psychological science, 25(11), 19871993.

Sali, A.W., Anderson, B.A., \& Yantis, S. (2014). The role of reward prediction in the control of attention. Journal of Experimental Psychology-Human Perception and Performance, 40, 1654-1664.

Tversky, A., \& Kahneman, D. (1981). The framing of decisions and the psychology of choice. Science, 211(4481), 453-458.

van Doorn, J., van den Bergh, D., Bohm, U., Dablander, F., Derks, K., Draws, T., ... \& Ly, A. (2019). The JASP guidelines for conducting and reporting a Bayesian analysis.

Vincent, B. (2011). Covert visual search: Prior beliefs are optimally combined with sensory evidence. Journal of Vision, 11(13), 25.

Publisher's note Springer Nature remains neutral with regard to jurisdictional claims in published maps and institutional affiliations. 